\title{
The Silent Hexagon: Explaining Comb Structures*
}

\author{
Tim Räz $z^{\dagger}$
}

October 26, 2020

\begin{abstract}
The paper presents, and discusses, four candidate explanations of the structure, and construction, of the bees' honeycomb. So far, philosophers have used one of these four explanations, based on the mathematical Honeycomb Conjecture, while the other three candidate explanations have been ignored. I use the four cases to resolve a dispute between Christopher Pincock (2012) and Alan Baker (2015) about the Honeycomb Conjecture explanation. Finally, I find that the two explanations focusing on the construction mechanism are more promising than those focusing exclusively on the resulting, optimal structure. The main reason for this is that optimal structures do not uniquely determine the relevant optimization leading to the optimal structure.
\end{abstract}

\section{Contents}

\begin{tabular}{lll}
\hline & Introduction & 2
\end{tabular}

2 The Honeycomb Conjecture 3

3 The Fejes Tóth Structure 4

\begin{tabular}{|lll}
\hline & The Liquid Equilibrium Hypothesis & 6
\end{tabular}

5 The Acoustic Resonance Hypothesis 10

6 Baker and Pincock on Comb Structures 13

\begin{tabular}{lll}
\hline 7 & Idealization & 15
\end{tabular}

$\begin{array}{lll}8 & \text { Optimization } & 17\end{array}$

*This is a pre-copyedited, author-produced PDF of an article accepted for publication in Synthese following peer review, DOI 10.1007/s11229-016-1014-3. The final publication is available at link.springer.com

†e-mail: tim.raez@posteo.de 


\section{Introduction}

Bees are mythical creatures. In our collective memory, they stand for fertility; the consumption of honey was taken to confer immortality; bees are a symbol for work ethic; they sacrifice their lives stinging their enemies; and they build beautifully shaped honeycombs. The scientific image of bees is no less fantastic than the myth. Bees perform dances in order to communicate the nature and location of resources; they are able to see ultraviolet light; and the bees' ability to build highly regular comb structures has received attention from scientists since antiquity. Darwin was famously interested in determining how exactly bees build their combs, because he took it to be an important test case for his theory of natural selection. He corresponded extensively on this matter, and wrote about it in the 'Origin' 1

Given the prominence of the question, it may come as a surprise that we still do not know how exactly the bees build their combs with the well-known hexagons on the surface. In the present paper, I will explore a number of mathematical and scientific attempts to explain the bees ${ }^{2}$ comb structure and its construction process. The four proposals I will consider range from the application of the mathematical Honeycomb Conjecture and an attempt to prove that the bees' cells are not optimal, to viewing the combs as a kind of liquid foam, and the bees exploiting acoustic phenomena to "tune" comb cells into the right shape.

The present paper has two goals. The first goal is to present, and discuss, these four candidate explanations of the structure, and construction, of the bees' honeycomb. So far, philosophers have used one of these four cases, which is based on the mathematical Honeycomb Conjecture, as a standard example of mathematical explanations. The explanation was proposed by Lyon and Colyvan (2008), and discussed in, e.g., Baker (2009, 2012, 2015), Baker and Colyvan (2011), Saatsi (2011), Lyon (2012), Pincock (2012), Tallant (2013), Lange (2013), Räz (2013), Baron (2014). However, the discussion has largely ignored three other candidate explanations and is, therefore, not adequately backed by science and mathematics. The exposition of the other three candidat ${ }^{3}$ explanations will improve the discussion by bringing the relevant science and mathematics to the attention of philosophers. I will present the pertinent mathematical and scientific results in some detail in sections 2 to 5 .

\footnotetext{
${ }^{1}$ See the entry 'The evolution of honeycomb' of the Darwin Correspondence Project, https://www.darwinproject.ac.uk/the-evolution-of-honey-comb, last retrieved June 23, 2015, and references therein.

${ }^{2}$ Not only certain kinds of bees (such as Apis mellifera), but also hornets (such as Vespa orientalis), and social wasps, build combs of high regularity.

${ }^{3}$ I will drop the qualifier "candidate" from here on. It should be noted that all four proposals are not (fully) accepted as actual explanations in science.
} 
The second goal of the paper is to use the four cases to shed light on pertinent philosophical debates. First, I use the four cases to resolve a dispute between Christopher Pincock (2012) and Alan Baker (2015) about the Honeycomb Conjecture explanation. I then analyze the role of idealization and optimization in the four cases. I find that while all four cases rely on idealizations and optimization results, the explanations that focus on the construction mechanism are more promising than those that exclusively focus on the resulting, optimal structure. The main reason for this is that optimal structures do not uniquely determine the relevant optimization leading to the optimal structure. The philosophical discussion can be found in sections 6 to 9 The main findings of the paper are summarized in the conclusion.

\section{The Honeycomb Conjecture}

The first explanation of the structure of the bees' honeycomb focuses on the most conspicuous feature of comb structures, the hexagonal shape of the comb cells; see figure 1. Aidan Lyon and Mark Colyvan (2008) propose that the mathematical Honeycomb Conjecture (HC) explains why the bees' honeycomb has the shape of the hexagonal tiling. Informally speaking, the HC says that the hexagonal tiling is the division of the plane into cells of equal area that minimizes perimeter, i.e., that the hexagonal tiling is the optimal tiling of the plane. If we add the biological premiss that the optimal tiling minimizes the necessary amount of building material -thus enhancing fitness- we get an explanation of the shape of the bees' honeycomb.

[FIGURE1. HEXAGONAL TILING]

Two objections to this explanation have been raised by Tim Räz (2013): First, the bees' honeycomb is three-dimensional, and nontrivially so. The bottom of cells is formed by three rhombi; see figure 2a for a geometrical representation. The bottom part of the cells cannot be explained if we only take the two-dimensional hexagonal shape of cells into account. This shows that an explanation of the shape of combs based on the $\mathrm{HC}$ is at least incomplete. The second, more tentative objection raised by Räz questions whether the HC is relevant to the explanation of comb structures at all. It may well be that some three-dimensional geometrical optimality result is relevant to the explanation of comb structures, but that it is not clear that the $\mathrm{HC}$ will be part of such an explanation. For example, the hexagonal tiling might be part of the boundary condition for the three-dimensional result, and thus be part of the explanans, not of the explanandum. In any case, it is not sufficient that the hexagonal tiling is part of the three-dimensional structure for the $\mathrm{HC}$ to be explanatorily relevant.

One might be tempted to think that the problem with the HC explanation is simply due to the fact that the $\mathrm{HC}$ is about two-dimensional structures, while actual comb structures are three-dimensional. However, this is not exactly right. Some real-world phenomena do have explanations based on two-dimensional results - think of the use of city street maps in order to navigate a city, or 
Euler's solution to the Königsberg bridges problem using a planar graph; see, e.g., Pincock (2007). The two-dimensional approach is inadequate in the present case because the bees' honeycomb has a three-dimensional structure that cannot be adequately captured in two dimensions.

The most important lesson we can learn from the -probably- unsuccessful explanation based on the $\mathrm{HC}$ is the following: If a mathematical structure $X$, such as the hexagonal tiling, is instantiated in an empirical system $x$, such as the bees' honeycomb, this does not mean that a mathematical result about $X$, such as the HC, also applies to the system $x$. If a mathematical structure $X$ is realized in an empirical system $x$, this realization $x$ can have a number of reasons that are not related to results about $X$.

\section{The Fejes Tóth Structure}

We have just seen that two-dimensional mathematical approaches to the bees' honeycomb are probably not the way to go. Is the bees' honeycomb the optimal solution to a three-dimensional optimization problem? Laszlo Fejes Tóth (1964) examined this question in a paper entitled "What the Bees Know and What They do not Know". Fejes Tóth's main goal is to determine whether the bees' honeycomb minimizes surface area, or whether other, superior designs exist. The biological motivation behind this question is a correspondence between the surface area of a given structure and the amount of wax necessary to build a honeycomb: less surface area means that less wax is necessary to build a honeycomb, which, in turn, increases fitness.

Fejes Tóth starts with a geometrical description of the bees' honeycomb. The structure is made up of one type of cell: hexagonal prisms with one open end and a bottom formed by three rhombi; see figure 2a (dubbed "Actual Honeycomb" in the following). These cells are arranged in two layers, oriented in opposite directions, with the hexagonal openings pointing outwards, and the rhombic caps meeting in the middle. In the next step, Fejes Tóth characterizes the type of structure to which we should compare the Actual Honeycomb. The cells should be arranged between two parallel planes, congruent (more specifically, only congruent convex polyhedra are considered), fill the space between the planes without overlap, and they should have an opening in exactly one of the two planes. The cells are arranged in two layers, with each cell belonging to exactly one of the two planes, via its opening. These features define a type of mathematical structure called honeycomb ${ }^{4}$ Finally, Fejes Tóth states the relevant optimization problem for honeycomb-type structures, the so-called first isoperimetric problem: Determine which honeycomb cell of given volume $v$ and given width $w$ minimizes surface area 5

\footnotetext{
${ }^{4}$ Fejes Tóth's formulation of honeycomb-type structures is but one possibility. In principle, there might be other relevant kinds of honeycombs. For example, honeycombs with different global structures, say, cells between two concentric cylinders, might be taken into consideration.

${ }^{5}$ The width $w$ is the distance between the two planes. The choice of width determines the
} 
[FIGURE 2a, ACTUAL HONEYCOMB AND 2b FEJES TOTH STRUCTURE]

Fejes Tóth then proves the main result of the paper: The Actual Honeycomb (figure 2a) is not the optimal solution to the first isoperimetric problem. The design of what I will call the "Fejes Tóth Structure", see figure 2b, is superior to the bees' design because of the cell bottom. The cell bottom of the Actual Honeycomb is based on the rhombic dodecahedron, while the cells of the Fejes Tóth Structure are based on a truncated octahedron. Fejes Tóth's cells have a smaller surface area for arbitrary widths. Fejes Tóth states the result as follows: "Instead of closing the bottom of a cell by three rhombi, as the bees do, it is always more efficient to use two hexagons and two rhombi" (Fejes Tóth, 1964 p. 473 , emphasis in original) ${ }^{6}$

Fejes Tóth calculates that the saving in surface area is less than $0.35 \%$ of the area of a cell opening - a very small gain in efficiency. Additionally, real cells are not perfectly regular, and we have not yet taken the thickness of walls into account. Therefore, the gain in efficiency might be negligible in practice. According to Fejes Tóth, the result is interesting within pure mathematics, but it does not warrant conclusions about actual honeycombs; he writes: "Under such conditions the above 'saving' is quite illusory" (Ibid., p. 473).

I see two ways of interpreting the fact that the Fejes Tóth structure is not realized by the bees. Possibility $(\mathrm{A})$ is that the bees are, in principle, able to construct combs of very high regularity, such that the small gain in efficiency could be realized, but this possibility is not realized because the gain in efficiency from using the Fejes Tóth structure is just not worth the effort. The energy necessary to improve the regularity might be higher than the gain in efficiency from the Fejes Tóth structure. If this is true, Fejes Tóth's optimization problem does not take the relevant optimization parameters into account, in that the energy that is necessary to enhance the regularity of structures is neglected. Possibility (B) is that the bees do not build the Fejes Tóth structure because it is beyond their abilities to build such a structure. One reason could be that they are not able to achieve the degree of regularity that is necessary to consistently realize the small gain in efficiency - evolution is not (yet) able to "see" the superior solution.

What are the consequences of these possibilities for the project of explaining comb structures? If (A) is true, Fejes Tóth's proposal is not actually explanatorily relevant in that it does not take all relevant optimization parameters into account, and a different formulation of the problem is needed. If (B) is true,

depth of cells: if the width is large compared to the volume, i.e., if $w \gg \sqrt[3]{v}$, which is true for actual honeycombs, then the cells will be deep orthogonal to the planes; otherwise, they will be shallow. Fejes Tóth also considers the optimization problem which lets the width of cells vary; this is the second isoperimetric problem. He shows that the bees' solution is not optimal with respect to this problem, but notes that it might be biologically sensible to let the cells be of a certain, fixed depth: a certain depth might be necessary for breeding purposes. Thus, the biologically relevant problem is the first isoperimetric problem.

${ }^{6}$ Fejes Tóth's is only a relative optimality result. The optimal solutions to both isoperimetric problems seems to be unknown. This is not surprising, because many three-dimensional optimization problems are unsolved. 
Fejes Tóth's proposal is explanatorily relevant - it shows that the bees' solution is suboptimal - but explanatorily incomplete in that we do not understand why the bees fail to build an optimal structure. Note that in Fejes Tóth's relative optimization result, the hexagonal tiling is not part of the explanandum, but of the explanans: The hexagonal tiling is assumed to be part of both structures under comparison.

\section{The Liquid Equilibrium Hypothesis}

\subsection{Dry Foams and Wet Foams}

Even if the two mathematical explanations of comb structures examined so far were not successful, mathematical optimization still seems like a promising approach to the problem. Unfortunately, many three-dimensional optimization problems that bear on the bees' honeycomb are still open 7 Fortunately, there are alternative approaches. One is a physical-mathematical framework that suggests an alternative path of inquiry: we can approach the problem experimentally. On this approach, the honeycomb is treated as a kind of foam 8 The mathematical connection between comb structures and foams is that in both cases, some sort of optimization is likely to be at play: the amount of wax for building a honeycomb is minimized because this is evolutionarily advantageous, while soap bubbles minimize surface area because this is energetically advantageous.

All mathematical structures we have seen so far can be classified as a kind of foam. Foams come in two varieties. Some foams have negligible liquid content, and the boundaries between bubbles are (almost) infinitely thin. These are called dry foams. Other foams have a non-negligible liquid content, and the bubbles do not have large common boundaries, but minimize surface energy one by one. These are called wet foams. The distinction between dry and wet foams carries over to optimal cellular structures with infinitely thin walls and cellular structures with thick walls, and we can classify optimization problems accordingly. In two dimensions, the Honeycomb Conjecture concerns the optimal dry foam of unit bubbles, while the analogue wet-foam problem is the circle-packing problem, i.e., the most efficient arrangement of unit circles in a plane 9 In three dimensions, the sphere-packing problem, or Kepler problem, is a wet-foam of unit bubbles (spheres); the analogue dry-foam problem, also known as the Kelvin problem, asks for the optimal dry-foam made of bubbles,

\footnotetext{
${ }^{7}$ For example, the optimal solution to the first isoperimetric problem formulated by Fejes Tóth is still open. Even problems that seem relatively easy are unsolved. For example, the optimal way of stacking cylinders in two layers is unknown; the same is true for the general "tin can stacking problem" in three dimensions; see Hales (2000, p. 440).

${ }^{8}$ The following discussion of different kinds of (mathematical) foams is based on Klarreich (2000) and Hales (2000). Weaire and Hutzler (1999) give an introduction to the physical aspects of foams.

${ }^{9}$ The solution to the circle packing problem is depicted in figure 3 part (a). See Szpiro (2003 ch. 3-4) for a detailed account of the history of the problem.
} 
or cells, of unit volume ${ }^{10}$

The foam problem relevant to comb structures is different from these problems because every cell has an opening; therefore, the honeycomb foam has to be bounded. We have already encountered a dry-foam problem that seems to capture the bees' honeycomb well, namely the first isoperimetric problem based on honeycomb-type structures defined by Fejes Tóth. What we have not yet examined is what happens when we add some liquid content to honeycombtype structures, i.e., when we consider the wet-foam analogue of Fejes Tóth's problem. Two physicists have explored this option: they produced a physical realization of the bees' honeycomb. The result of the experiment reveals a connection between close-packing and surface minimization problems, or dry foams and wet foams.

\subsection{Physical Foams}

Denis Weaire and Robert Phelan (1994) report their experiment in a paper entitled "Optimal design of honeycombs". After summing up the results by Fejes Tóth, they describe how they reproduced honeycomb-type structures with soap bubbles. They introduced equal-sized bubbles of a liquid solution between two glass plates, such that the bubbles formed a double layer of cells. This is a bounded foam. Weaire \& Phelan observe that the two layers formed an array of cells with hexagonal openings. Their main finding is that the design of the cell bottoms changes if the liquid content of the foam is varied:

[In the case of a] foam of low liquid content [...] which corresponds to Toth's geometrical picture, we observe the structure proposed by him [see figure $2 \mathrm{~b}$. If we wet the foam by the addition of more liquid, the junctions between films are thickened to form what are called Plateau borders. The conditions under which surface energy is to be minimized are accordingly changed. What happens as the foam is progressively wetted is quite dramatic. At a certain point the Toth structure becomes unstable and there is a sudden switch to the configuration favoured by the bees [see figure 2a. Such a switch also takes place in the reverse direction, as liquid is removed. (Weaire and Phelan, 1994)

The experiment, then, yields three main findings. First, the Fejes Tóth Structure (figure 2b) is realized as an actual dry foam. Second, the Actual Honeycomb (figure 2a) is realized as an actual wet foam. Third, and most surprising, the structures can be transformed into each other by adding or subtracting liquid content. Weaire \& Phelan's finding provides a physical verification of the fact that both the Fejes Tóth Structure and the Actual Honeycomb are optimal

\footnotetext{
${ }^{10}$ The Kepler problem was solved by Hales, and only the Kelvin problem remains open. For a long time, the candidate solution was a structure proposed by Kelvin, but in 1994, Denis Weaire and Robert Phelan found a counterexample to Kelvin's structure, now called Weaire-Phelan structure.
} 
solutions: the Fejes Tóth Structure is the optimal solution to the dry-foam version of the problem, while the Actual Honeycomb is the optimal solution to a wet-foam version 11

\subsection{Biological Foams}

The realization of the mathematical honeycomb structure as a physical foam does not establish that the process leading to biological comb structures is related to the equilibrium processes at play in the physical foam. In order to establish this, empirical studies of comb structures are necessary. Such studies have been carried out. They show that the bees' honeycomb might be an actual foam in that the construction of the honeycomb relies on a liquid equilibrium ${ }^{12}$ Pirk et al. (2004) examined the construction process of the bees' honeycomb. The bees do not construct the honeycomb cells entirely "by hand"; rather, they build a rough framework of cells, which is then melted into its final shape:

The structure of the combs of honeybees results from wax as a thermoplastic building medium, which softens and hardens as a result of increasing and decreasing temperatures. It flows among an array of transient, close-packed cylinders which are actually the self-heated honeybees themselves. (Ibid., p. 350)

The bees raise their body temperature to over $40^{\circ} \mathrm{C}$ in a coordinated process - they work simultaneously in neighboring cells in both layers - and the cells get their characteristic hexagonal shape through a thermodynamic process: "the comb structure is a result of a thermoplastic wax reaching a liquid equilibrium" (Ibid., p. 352). These empirical findings suggest that biological comb structures are in fact built on the basis of a liquid equilibrium process ${ }^{13}$

However, the findings by Pirk et al. (2004) have been contested. Daniel Bauer and Kaspar Bienefeld (2013) question whether a liquid equilibrium is at play as claimed by Pirk et al. and Hepburn et al. . Bauer and Bienefeld examined the construction process the combs of European honeybees (Apis mellifera) using infrared and thermographic video observations. They observed, first, that the bees formed the wax with their mandibles, using their legs for stabilization and to apply the necessary force. Second, the temperature of the wax never

\footnotetext{
${ }^{11}$ The experiment does not constitutes a mathematical answer to the mathematical dryfoam and the wet-foam problems. A mathematical proof is missing in both cases.

${ }^{12}$ Hypotheses linking the bees' honeycomb to soap bubbles via an equilibrium process have been around for some time; see e.g. Klarreich (2000 p. 159). The biological results reported below were unknown to Weaire \& Phelan in 1994.

${ }^{13}$ Pirk et al. also found that the cell bottoms, or closings, are not in fact formed by three rhombi (see figure 2a for the rhombic design), but spherical. This finding about the cell bases, however, has been contested by Hepburn et al. (2007). They examined moulds taken from newly constructed as well as from old cells, and found that while old cells indeed have spherical bases, newly-constructed cells have rhombic bases. Pirk et al. made the mistake of taking moulds exclusively from old cells. This finding is an important part of the bigger picture: if the cell bases were spherical, this would indicate that not all parts of cells were constructed by thermal equilibrium; the finding by Hepburn et al. shows that the thermal construction can explain the structure of the whole cell.
} 
rose above $37.8^{\circ} \mathrm{C}$, and the thorax temperature of builder bees never rose above $39.6^{\circ} \mathrm{C}$, well below the $40^{\circ} \mathrm{C}$ found by Pirk et al., which is necessary for the liquid equilibrium: "[t]he wax was compact and did not enter the liquid equilibrium state at any of the building temperatures observed" (Bauer and Bienefeld, 2013, p. 48). Bauer and Bienefeld write that heating up the wax may contribute to its plasticity and thereby may facilitate its (mechanical) shaping.

David Bergman and Jacob Ishay (2007) question whether the Liquid Equilibrium Hypothesis is relevant in all cases of comb construction. Most importantly, the material used in the construction of combs of Oriental hornets is incompatible with the construction process as proposed in the Liquid Equilibrium Hypothesis. Oriental hornets use gravel and fibers, mixed with their saliva, to construct their comb. It has been empirically tested that the melting point of this cement-like material is over $100^{\circ} \mathrm{C}$ - above the temperatures measured by, e.g., Pirk et al. (2004). Thus, at least some comb structures are not shaped by a liquid equilibrium. However, this does not rule out that a kind of foam, or physical equilibration, is relevant in the construction of other combs.

\subsection{Interlude: What is Optimized?}

If the Liquid Equilibrium Hypothesis is correct, the result by Weaire \& Phelan raises the question as to what kind of optimization is at play in comb construction. Weaire \& Phelan establish a relation between two kinds of problems, viz. tiling problems and packing problems. Tiling problems ask for a division of space into cells such that only negligible parts of the space are left over - dry foams -, while packing problems ask for the most efficient packing of bubbles of a given shape in space - wet foams. The bees realize the Actual Honeycomb, a sort of wet foam, not the Fejes Tóth Structure, a dry foam. Thus, the primary optimization problem might be a three-dimensional packing problem, not a three-dimensional tiling problem 14 Accordingly, the amount of wax or building material used in construction might not be the most important optimization parameter; rather, the primary problem might be the optimal storage of bees themselves (or their larvae) so as to preserve heat.

The construction of comb cells might be a two-step procedure that mirrors Weaire \& Phelan's experiment. In the first step, the bees build cells that make it possible to pack themselves as closely as possible in two layers - a close-packing of cylinders. In the second step, they subtract wax from the close-packed cylinders by melting away superfluous wax. The melting does not yield an actual dry foam, as the Fejes Tóth structure is not realized, but maybe, if the bees continued to melt away wax, the structural transition from the Actual Honeycomb to the Fejes Tóth Structure might occur, just as in Weaire \& Phelan's experiment 15

\footnotetext{
${ }^{14}$ The idea that the primary optimization might concern packing has been around for some time; see Pirk et al. (2004) for some useful references.

${ }^{15}$ Other parameters besides optimized packing and tiling are relevant to comb construction. For example, the structure has to be sufficiently stable; see Weaire and Hutzler (1999, p. 167). The actual honeycomb might not reach the point of structural transition realized in the
} 


\section{[FIGURE 3. CIRCLE PACKING AND VORONOI CELLS]}

One part of the combs, however, does exhibit a dry foam structure. The twodimensional cross-section of comb cells is a hexagonal tiling, i.e., an optimal dry foam. The corresponding optimal wet foam consists of circles, each surrounded by six circles; see figure 3 part (a). Just as in Weaire \& Phelan's experiment, there is a connection between the optimal packing of circles, and the hexagonal tiling. If we start from the circle packing and divide the space between circles such that each point is assigned to the closest circle, we arrive at the hexagonal tiling: the points lying exactly between two or three circles are the points of the hexagonal grid; see figure 3 part (b). Thus, some parts of the cells do undergo the structural transition (cross-section), while others (cell bottoms) do not.

\section{The Acoustic Resonance Hypothesis}

The fourth explanation of comb construction describes a mechanism that makes it possible for bees, hornets, and wasps to construct combs "by hand" in a process of trial and error. Bergman and Ishay (2007) and Kadmon et al. (2009) propose that hornets, wasps, and bees use acoustic resonances in the construction of their combs. Resonance frequencies can be used to detect whether neighboring cells have a similar cross sectional area. They can also be used to detect whether a single cell has the symmetry of a hexagon.

\subsection{The Explanandum}

The description of the explanandum by Bergman and Ishay (2007) encompasses observations about comb structures as well as the construction process. The structure of a comb is highly symmetrical in the lateral cross section, i.e., in a plane parallel to the openings of the cells. The symmetry is that of a hexagonal tiling of the plane; see figure 1. The depth of the cells, however, varies considerably, as does the shape of openings and bottoms, especially towards the edge of a comb. Also, while the lateral symmetry is high within one comb, different combs in the same hive can have different sizes ${ }^{16}$ The explanandum, then, is the cell's lateral hexagonal symmetry. This symmetry stands in contrast to the lack of symmetry in the non-lateral dimension. The explanation should take into account that the relative size of cells can vary, but that cells in one comb are of the same size and hexagonal shape ${ }^{17}$ Turning to comb construction, the hexagonal cross section of cells emerges only gradually. The first cell of a comb is usually approximately round, with six corners roughly in the right places; only when the surrounding cells are added does the hexagonal shape start to show. Then, the precision of construction seems to be correlated with the degree

\footnotetext{
Weaire-Phelan experiment because stability stands in the way.

${ }^{16}$ The biological reason for this variation is that the cells are built for breeding different kinds of hornets. Breeding drones and queens requires larger cells than breeding smaller working hornets.

${ }^{17}$ Note that if the explanandum is the lateral symmetry of cells, it could be thought that the $\mathrm{HC}$ is relevant to the explanation. We will see below that it is not.
} 
of cooperation in construction; if a small group of Oriental hornets construct a comb, the precision decreases. Finally, Oriental hornets usually build their hive in dark caverns; thus, they do not use visual information during construction. The explanation should take these observations about the construction process into account.

\subsection{The Explanans}

The explanation is based on physical models from room acoustics ${ }^{18}$ Comb cells are modeled as straight ducts with constant cross section in the $x-y$ plane and point symmetry along the $z$ axis. The acoustics of this kind of room can be studied using acoustic waves that are periodic in time, so-called room modes. The simplest case is a duct with rectangular cross section, which I will use as an illustration; see figure 4 .

[FIGURE 4. ROOM MODES]

In straight ducts, we can distinguish different kinds of modes. So-called lateral modes are aligned with the cross section of the duct. Lateral modes have two important properties that are relevant to cell construction. First, lateral modes are non-propagating, while non-lateral modes will propagate in the direction of the axis of the duct. Due to this property, lateral modes can store energy and have a long lifetime, while propagating modes lose energy to the environment. Thus, if the air in a duct is excited at the frequency of a lateral mode, there will be a strong "response" from that lateral mode at that frequency. These so-called eigenfrequencies can be used to distinguish the different lateral modes. Second, the frequencies of different lateral modes are on a discrete spectrum; see figure 4 for a lateral mode of a rectangular cross section. The different lateral modes of a duct with rectangular cross section are distinguished by the number of nodes of constant pressure - two nodes in the $x$ and two nodes in the $y$ direction in the case of figure 4. Thus, each lateral mode is separated from every other lateral mode and has a distinct eigenfrequency.

Constructing Similar Cells The lateral modes can be used to detect whether different cells have the same size. If two neighboring cells have the same cross section, then the lateral modes of the two cells will coincide, and they will resonate at the same frequency. If, however, their sizes are slightly different, then the lateral modes of the two cells have slightly different frequencies. The frequencies will interfere and produce so-called beats, which arise from the superposition of the two modes. These changes in beat frequency can be used to "tune" the cells like an instrument. A decreasing beat frequency means that the frequencies of the lateral modes are closer, which means that the size of the cells has become more equal. This leads to the following "construction recipe" for assimilating adjacent cells. First, measure the beat frequency of two cells. Then try to make the cells more similar. Finally, measure the beat frequency again. If the beat frequency is increased, the cells have become more dissimilar, and the

\footnotetext{
${ }^{18}$ See Pierce $\sqrt{1989}$ sections $\left.6.5 ; 7.1\right)$ for a detailed account of the relevant models.
} 
changes should be reversed. If the beat frequency has decreased, the cells have become more similar, and the direction of change should be continued. This construction recipe can be used by one or several hornets.

Constructing Symmetric Cells The lateral modes of a cell can also be used to determine whether the cell's cross section has the symmetry of a regular polygon ${ }^{19}$ In order to explain this in more detail, we need a mathematical characterization of the relevant models from room acoustics. The lateral room modes of a duct with constant cross sections can be found by solving a twodimensional Helmholtz equation with Neumann boundary conditions. The lateral modes correspond to eigenfunctions of this problem, and the frequencies of the room modes correspond to the eigenvalues of these eigenfunctions ${ }^{20}$ Some of these eigenvalues are twofold degenerate, i.e., one eigenvalue is associated twice with a particular eigenfunction. The twofold degeneracy of eigenvalues, in turn, can be used to distinguish between all cross sections that have a socalled two-dimensional irreducible representation, and those that do not ${ }^{21}$ The cross sections that have this property are the regular (convex) polygons and the circle as a limit case. The symmetry group of these polygons is the dihedral group. Importantly, the twofold degeneracy does not uniquely single out the hexagon among all possible cross-sectional shapes of a cell, because it is a symmetry property that is detected, not the hexagonal shape itself, and other cross sections besides the hexagon do have this symmetry. However, most regular polygons are not suitable for comb construction. If we assume that the cells have a constant cross section, and that the cross section is a tiling, then only the equilateral triangle, the square, and the hexagon are viable candidates. Of these three shapes, the hexagon is the most efficient, because its perimeter is much smaller than that of the square or the equilateral triangle ${ }^{22}$ Thus, if it is

\footnotetext{
${ }^{19}$ The following is mainly based on Kadmon et al. (2009).

${ }^{20}$ Note that if the boundary is hexagonal, this problem does not have closed-form analytic solutions and has to be solved numerically. The numerical procedure yields eigenvalues; see Kadmon et al. (2009, table 1).

${ }^{21}$ Representation theory studies symmetries by examining how a group acts on linear vector spaces. An irreducible representation is the fundamental building block of this action. The determination of symmetry works as follows. According to representation theory, the number $p$ of components of an irreducible representation has to be at least as big as the number $s$ of distinct eigenvalues of an operator associated with a system, if the system has the symmetry group of this representation $(s \leq p)$ (this is an application of Schur's lemma). If the number of distinct eigenvalues $s$ is bigger than the number $p$ of the components of an irreducible representation, this means that the system does not have the symmetry group of this representation. If, however, $s$ matches $p$, we have evidence that this is the right symmetry group. In the current situation, the connection between frequencies and symmetry is as follows. Assume we observe $s$ distinct frequencies. Each frequency corresponds to an eigenvalue. If two frequencies of the cell coincide, we have one eigenvalue associated with a lateral mode, i.e., $s=1$, which means that we have evidence for $p=1$, such that the two-dimensional space has an irreducible representation with one component, i.e., the irreducible representation itself is two-dimensional. Thus, the cell has a symmetry with a two-dimensional irreducible representation. A useful account of the mathematics can be found in Sternberg (1994. Ch. $2-3)$.

${ }^{22}$ This fact has been known since antiquity; its first statement is usually attributed to Pappus; see Hales (2001).
} 
adaptive to build cells with a minimal cross section, and if there is a preference for cells with a regular, symmetric cross section, then the hexagonal design is the most efficient choice. The symmetry of the hexagon can then be used to detect how close the cross section of a cell is to a hexagon. Long story short: The animals know how to make cells that are approximately hexagonal, and the symmetry helps them to perfect their shape.

Checking the Explanation's Adequacy The above account constitutes a candidate explanation of how comb structures are built by bees, wasps, and hornets. However, more is needed to ensure that this is an actual explanation. First, the underlying physical model is quite idealized, and it is necessary to check whether the idealizations can be neglected. Bergman and Ishay (2007) base all their calculations on a model with constant, circular cross section. Further idealizations are that the duct is infinitely extended along the axis of the duct, that the air is a perfect fluid, and that the walls are smooth and perfectly rigid. Bergman and Ishay write that their model is nevertheless sufficiently similar to provide rough quantitative estimates for the acoustic frequencies involved in construction. Kadmon et al. (2009) consider various de-idealizations of the first model ${ }^{23}$ They find that while some idealizations cannot be adequately evaluated for the time being, none of those that can be evaluated speaks against their account. Second, it has to be checked whether the resonance frequency derived from the model is detectable in de-idealized scenarios. Most importantly, the relevant frequency has to be clearly separate from frequencies associated with propagating modes and from other resonance frequencies. Kadmon et al. $(2009$, sec. IV) argue that this is the case for the frequency of the lowest non-zero lateral room mode. Third, it has to be checked whether acoustic resonances are accessible to the animals. Are bees, or hornets, able to produce, and hear, the required frequencies? Bergman and Ishay (2007, p. 1781) note that honeybees have been observed to produce ultrasonic waves with the right frequencies.

\section{Baker and Pincock on Comb Structures}

The explanation of honeycomb structures based on the Honeycomb Conjecture has been one of the standard cases in the philosophical debate on mathematical explanations. Taking the other approaches to comb structures into account will hopefully lead to a more nuanced discussion of the role of mathematics in application. Here I will take first steps towards this goal.

In a recent paper, Alan Baker (2015) discusses the use of different mathematical results in application to an empirical system. Baker defends the thesis that if we apply mathematical results, there is a tradeoff between the strength of the mathematical result and ontological commitments concerning empirical systems: stronger mathematical results reduce ontological commitments. He argues that

\footnotetext{
${ }^{23}$ Numerical calculations provide the eigenvalues of the physical model with a hexagonal cross section; this makes it possible to get approximations of the acoustic frequencies that may be used in comb construction. Kadmon et al. also address the other idealizations.
} 
the bees' honeycomb is such a case. In an earlier contribution, Christopher Pincock (2012) has pointed out that instead of using the strong Honeycomb Conjecture to explain the bees' honeycomb, we might use the weaker result that the hexagonal tiling is the most efficient shape among regular polygons. Pincock argues that if we want to use the weaker result, we need an additional justification as to why the bees are constrained to regular polygons in optimizing the honeycomb. One such reason is that "[the regular polygons] might be easier to 'program' into the genetic code of the bees than an intricate irregular polygon pattern" (Ibid., p. 213). In reaction to this, Baker grants that if there were real, biological reasons for using the weaker optimization result, Pincock's argument would apply, but that, currently, there are no such reasons. Therefore, Baker contends, Pincock's argument is speculative, and the use of the stronger Honeycomb Conjecture is warranted, because this reduces a commitment to concrete physical constraints, such as the commitment to regular polygons.

The present study shows that there are, in fact, biological reasons as to why the result about the optimality among regular polygons is relevant, and not the more general Honeycomb Conjecture. According to the Acoustic Resonance Hypothesis, the animals use the symmetry property of regular polygons in the detection of these shapes as opposed to all other (irregular) polygons. Among the regular polygons, the hexagon is selected because this is adaptive, either by minimizing the amount of building material, or because it results from a close-packing. Acoustic resonances are only suitable to solve this (restricted) optimization problem. Thus, there is no need to speculate about this case; there are real reasons why the weaker mathematical result is relevant.

We can also learn a systematic lesson about Baker's thesis from the present case. We saw that the construction mechanism plays an important role in the tradeoff between the strength of mathematical results and ontological commitments. Take, first, the Liquid Equilibrium Hypothesis. If this is the actual construction mechanism, then the optimization is realized on a molecular level, which does not place many restrictions on the kind of mathematical result that can be realized, in that the animals do not need to "see", or be "programmed" for, the optimal solution. They only need to be able to facilitate the optimization on the molecular level. If, on the other hand, the underlying mechanism is that of the Acoustic Resonance Hypothesis, then the animals realize the optimal structure mechanically. This makes it necessary for the animals to be able to detect, or "see", the optimum, say, via a symmetry property.

Thus, the weaker result might be preferable exactly because it is weaker: the set of alternatives under consideration has more structural properties, which, in turn, makes the fewer structures captured by the weak result epistemically accessible. Baker's thesis of a tradeoff between stronger mathematical results and less ontological commitment may be true as far as it goes, but stronger mathematical results usually rely on less structure, which may be an epistemic disadvantage. The less structure is needed to establish a mathematical result, the less structure is left to be detected. I return to the relation between the two optimization results in section 8 below. 


\section{Idealization}

The use of idealizations is a common theme of all four cases. Idealizations are modeling assumptions that are, strictly speaking, wrong of a target system ${ }^{24}$ The four explanations differ in the way in which idealizations are approached; this will emerge from a an examination of the role of idealizations in all four cases.

One problem of the Honeycomb Conjecture explanation is that it neglects the essentially three-dimensional nature of actual comb structures. This can be interpreted as an idealization that turns out to be unjustified - if the comb structure is modeled in two dimensions, an important part of the structure is necessarily left out. If we focus on a different explanandum, such as the lateral cross-section of a comb, the $\mathrm{HC}$ cannot be rejected as irrelevant. It is not its idealized nature that leads to the irrelevance of the HC in the Acoustic Resonance Hypothesis, but the fact that it is incompatible with the construction principle, as I argued above.

The final judgement as to the adequacy of the Fejes Tóth structure depends on the reason why the Fejes Tóth structure is not realized by the bees, as discussed in section 3 . If possibility (A) is correct, such that the gain in efficiency is simply not worth the effort, then Fejes Tóth's result uses an inadmissible idealization in that it neglects one crucial optimization parameter, the energy necessary to achieve a high degree of accuracy. If possibility (B) is true, then the Fejes Tóth structure might be realized some time in the future, and the idealizations turn out to be justified. It is unclear how we could decide between these two possibilities for the time being. However, Fejes Tóth's result does not conclusively establish that the bees' honeycomb is suboptimal. Note that there are other idealizations at play in Fejes Tóth's proposal; for example, both the Actual Honeycomb and the Fejes Tóth Structure are assumed to be infinitely extended between the two planes. The idealized system that is infinitely extended between the two planes can be interpreted as approximating a large but finite system, using the distinction between idealized systems and approximation assumptions emphasized by John Norton (2012). The infinite nature of the system does not have properties that are inconsistent with the finite case that is approximated. The idealization helps simplifying the problem, and it appears to be harmless.

The Liquid Equilibrium Hypothesis uses idealizations as well, but the idealized model is not mathematical. Rather, the physical foam produced in Weaire \& Phelan's experiment can be interpreted as a physical model of biological comb structures. The physical foam is an idealized model of biological foams because biological foams have a different constitution - the physical foam consists of soap film, which is not the case in biological combs. If we want to use the physical foam as a model of biological combs, we have to make sure that the latter shares a relevant property with the physical foam, viz. that the biological comb

\footnotetext{
${ }^{24}$ There is now an extensive philosophical literature on idealizations; see Frigg and Hartmann (2012) for a useful discussion of idealizations in the context of scientific models.
} 
is shaped by a liquid equilibrium. The physical model leads to specific predictions about features that the target systems, biological comb structures, need to have in order for the Liquid Equilibrium Hypothesis to be an explanation: the combs have to be amenable to a thermal equilibrium; the animals need to be able to heat the building material to the melting point; and so on. The result of testing these predictions is that, at least in some biological comb structures, the Liquid Equilibrium Hypothesis is not true, because the construction mechanism cannot be realized: the comb material is unsuitable for a thermal equilibrium in hornet combs, and it is questionable for honeybees. The physical foam is not an actual model of biological foams in these cases. This does not exclude that the Liquid Equilibrium Hypothesis is true in other biological comb structures.

In the Acoustic Resonance Hypothesis, Bergman and Ishay (2007) and Kadmon et al. (2009) explicitly discuss the idealizations of the various models. They examine a sequence of physical models from room acoustics, starting with ducts with circular cross section in Bergman and Ishay (2007), then turning to ducts with hexagonal cross section, and finally examining models with energy dissipation and non-rigid walls in Kadmon et al. (2009). Why exactly do the scientists examine this sequence of models? One reason why Bergman and Ishay (2007) only consider the model with circular cross section is that the model with a hexagonal cross section can only be examined numerically and in approximation. The model with a circular cross section is mathematically more tractable, and it is, therefore, sensible to start with this simple model. There is a clear tradeoff between mathematical tractability and the degree of idealization.

Prima facie, it could be thought that the goal of this sequence of models is to construct the real, final model that will replace the previous, more idealized models, so as to base the explanation on the last member of the sequence. The idea that scientists aim at de-idealizing models has been questioned in the debate on scientific models; see, e.g., the contributions by Nancy Cartwright (1983, 1989). The main reason why de-idealizing is seen as problematic is that finding a de-idealized model will usually not be possible, and that scientists do not usually try do construct one de-idealized model to replace more idealized models.

I take the Acoustic Resonance Hypothesis to rely on de-idealizations, but they are of a different kind than those usually discussed and criticized. The goal of Bergman and Ishay (2007) and Kadmon et al. (2009) is not to construct one de-idealized model, but to check whether the various idealizations made in the first model are harmful. The de-idealized models are not taken to replace the more idealized models; they are supposed to help checking separate idealizing assumptions. The rationale behind the more de-idealized models is not so much to find the final explanation, but to validate the explanation that we already have, to the extent that this is possible. Thus, the scientists do not de-idealize the whole model, they merely check whether some of the idealizing assumptions are harmful. This is a more modest goal than constructing a full, de-idealized model.

In sum, all four explanations make use of idealizations. However, the explanations differ in how well they are able to deal with idealizations. The HC 
explanation is likely to be inadequate, Fejes Tóth's proposal is in limbo; it is not clear how to check whether the proposal is an actual explanation or not. In the case of the Liquid Equilibrium Hypothesis and the Acoustic Resonance Hypothesis, on the other hand, it is clear how to check whether the idealizations are justified, and both have been subjected to empirical tests. This has led to a clear rejection of the Liquid Equilibrium Hypothesis in some cases. The Acoustic Resonance Hypothesis is in need of further testing.

\section{Optimization}

The use of optimization results is also an important aspect of all four cases. The first case uses the Honeycomb Conjecture to explain the hexagonal shape of comb structures. Fejes Tóth's structure is supposed to show that the bees' honeycomb is not optimal by formulating an optimization problem, and then showing that there is a structure that fares better than the biological comb. The Liquid Equilibrium Hypothesis exhibits yet another way in which the hexagonal tiling might arise, viz. as the result of a two-stage optimization process involving the optimal packing of circles. The Acoustic Resonance Hypothesis, finally, proposes that the hexagonal tiling is detected via its symmetry property. As a result, this proposal relies on a weak optimization result about regular tilings of the plane.

There are thus four kinds of optimization in the four proposals - and the hexagonal tiling features in all four proposals. We face a serious case of underdetermination: the hexagonal tiling is a "multiply optimal structure". This shows that even if an empirical structure is the result of some optimization, this does not establish which one of a range of possible optimizations is responsible for the optimal outcome, because structures can be the solutions to more than one optimization problem. In addition, the Liquid Equilibrium Hypothesis establishes that the hexagonal tiling might arise due to a different kind of optimization: not an optimal tiling, but an optimal packing. Empirical studies are needed in order to decide whether the biologically relevant optimization parameter is minimization of building material, or close-packing and subsequent optimization of space.

The fact that a mathematically optimal structure, such as the hexagonal tiling, can be instantiated for a number of reasons, which need not be related to optimization, has been discussed in the debate on mathematical explanation and optimization. For example, Christopher Pincock (2014) writes that in optimization explanations - such as the explanation of Plateau's laws - a structural correspondence between mathematical and empirical structures is not sufficient for explanation. Otherwise, we would have to accept that we can explain, say, the shape of a manufactured plastic sphere using the fact that this is the surfaceminimizing shape of a single, three-dimensional cell. Pincock points out that there needs to be an "appropriate link" between the explaining property, and the system that instantiates the explanandum property. The underdetermination problem pointed out here shows that even if a structure is the outcome 
of some optimization mechanism, we cannot conclude that it is the outcome of one particular kind of optimization. It is not sufficient to require that some kind of optimization is at play in the empirical structure for a mathematical optimization result to be applicable.

The Acoustic Resonance Hypothesis has a further perplexing upshot: an empirical structure can be the result of a particular kind of optimization, but this does not imply that the strongest mathematical result about this kind of optimization helps explaining the empirical structure. We know that the hexagonal tiling is the optimal tiling of the plane from the Honeycomb Conjecture, and, according to the Acoustic Resonance Hypothesis, the hexagon is in fact picked out because it is an optimal tiling. However, the hexagon is not selected because it is optimal according to the $H C$, but because it is optimal compared to the square and the triangle. This result is much weaker than the $\mathrm{HC}$, which subsumes the result for regular polygons. Nevertheless, it is the result on regular tilings that is relevant here.

However, this might seem problematic for the following reason. The Honeycomb Conjecture mathematically encompasses the result about regular polygons: If the hexagon is the best possible tiling, then, because it is regular, it is also the best regular tiling. Why, then, is the HC not applicable, while the result about regular tilings is applicable? I grant that the HC is the mathematically stronger result: it subsumes the result about regular polygons, as far as geometric optimality is concerned. However, in the context of empirical application, geometric structure is not all that is relevant. A structural correspondence between mathematical and empirical structure is not sufficient for explanation, as Pincock pointed out. If we want to apply a mathematical optimality result to an empirical structure, this structure needs to be realized in the right kind of way: it needs to be produced by the right kind of empirical optimization process. If the Acoustic Resonance Hypothesis is correct about the empirical optimization process at play, then it is not the $\mathrm{HC}$, but the result about regular polygons that is relevant, because the regular polygons have a structural property, symmetry, which is used by the animals to detect the regular polygons. The animals "exploit" the epistemic advantage of this additional structural property of regular polygons in the detection of the optimal shape; consequently, only the weaker optimality result applies here. Note that the symmetry property used to detect the regular polygons has nothing to do with optimization a priori 25

\footnotetext{
${ }^{25} \mathrm{An}$ anonymous referee has pointed out that the HC might play an explanatory role in the Acoustic Resonance Hypothesis after all: The HC shows that using the optimal regular polygon is sufficient, because it is the also the global optimum. I agree. However, this is an explanatory relation between two mathematical results, not between the explanandum in question, which is an empirical structure, and a mathematical result. An explanatory relation between the two mathematical results does not imply that the $\mathrm{HC}$ explains anything about empirical comb structures; for the latter, additional empirical considerations come into play.
} 


\section{Structure vs. Construction Mechanism}

In this section, I turn to the most important systematic difference between the first two and the second two cases: the former focus on the resulting geometrical structure, while the latter focus on the construction mechanism leading to the structure. The first two explanations do not pay attention to the way in which the optimization is realized; they conform, roughly, to "Optimality Explanations" as advocated by Collin Rice (2013). According to Rice, optimality explanations are often highly idealized and do not cite any casual processes as part of the explanation. The latter two, on the other hand, seem to conform to ideas formulated in the New Mechanistic account of explanations, initiated in Machamer et al. (2000) and Glennan (2002). These explanation rely on the (causal) processes that lead to the outcome of a certain geometrical structure.

The above survey of the four cases suggests that the approaches focusing on the construction mechanism have a better chance at being actual explanations; at least, they are easier to evaluate. The Honeycomb Conjecture explanation is, in all probability, inaccurate, and it is not clear whether the Fejes Tóth structure is relevant to comb construction. On the other hand, the Liquid Equilibrium Hypothesis can be seen to be clearly inadequate in some cases, and the Acoustic Resonance Hypothesis is the most likely candidate for an actual explanation.

One reason for this difference is the underdetermination problem pointed out in the previous section. If one wants to explain the final structure using a result from mathematical optimization theory, one may have to choose between several optimization problems that yield the same structure. This makes it difficult to evaluate an explanation that relies on the resulting structure only. Taking the construction mechanism into account has the advantage of yielding empirically testable consequences in a more direct manner. The Liquid Equilibrium Hypothesis requires a liquid equilibrium; the Acoustic Resonance Hypothesis requires a mechanical, trial and error construction process which is guided by resonances; and we can check whether the empirical systems in question have the required features. The empirical consequences are one step further away if we focus in the resulting structure - the mathematics is silent on how exactly the optimization is realized. This is a serious drawback if we are after an actual explanation.

It might be objected that the comparison of the two approaches to comb structures is unfair, because the two camps are just after different explananda: the construction process and the outcome of that process are two different phenomena. However, this is not quite right. In the Liquid Equilibrium Hypothesis, a mathematical optimization result for comb structures is missing, but Weaire \& Phelan indicate that a wet-foam formulation might be an adequate formulation of the problem. In the case of the Acoustic Resonance Hypothesis, a long-known mathematical optimization result for regular polygons is relevant. In both cases, mathematical optimization and the resulting structure play a substantive role.

The contrast between an explanation of the resulting structure vs. an explanation of the construction mechanism should not be construed as one between an abstract, idealized explanation of a structure, and a causal explanation that 
gives us the history of the genesis of that structure in full. Rather, explanations of the construction mechanism focus on the construction principles of the structures. They tell us, in general terms, how the structures came about, by isolating the principle used in construction, be it a liquid equilibrium or a mechanical trial and error process guided by acoustic resonances. These explanations use idealizations as well. However, the fact that the construction principle is built into the explanations makes it easier to check whether the idealizing assumptions lead us astray, or are harmless.

The two proposals taking the construction mechanism into account fare better not because they provide us with a better understanding of the phenomenon in question, or because every explanation has to cite causes or causal processes with necessity. The reason why they are superior is that even if a structure is optimal according to some optimality result, we have to make sure that the structure arises because the right sort of optimization is actually at play in the system. Otherwise, we might not be dealing with an actual explanation. If an optimum is underdetermined, we cannot avoid examining the process leading to the optimum to get rid of the underdetermination problem.

In sum, the evaluation of the two different kinds of approaches shows that, as a matter of fact, the approaches that take the construction process as a starting point fare better in terms of empirical adequacy. The present study can be read as a cautionary tale about a "Moving Beyond Causes" approach, i.e., an almost purely mathematical approach to optimization, as advocated by Rice (2013). Of course, it can be questioned how general the lesson is we can learn from the present study. However, I have stated systematic reasons why the focus on the construction mechanism is advantageous, namely the underdetermination of the optimization problem by the structure, and the fact that the construction mechanism yields empirical consequences more readily.

\section{Conclusion}

The most important systematic lessons from the philosophical discussion are the following. First, I critically examined Alan Baker's thesis that, in some mathematical explanations, we can reduce ontological commitment if we use stronger mathematical results. I argued that using stronger mathematical results also means that we use "less structure", which makes the structure in question epistemically less accessible. The Acoustic Resonance Hypothesis is a case in point. The bees use a symmetry property in construction that would not be available if the Honeycomb Conjecture were relevant to the construction of the bees' honeycomb. Second, I argued that a form of de-idealization is at play in the Acoustic Resonance Hypothesis. However, the goal of de-idealization is not so much to find the final model, and explanation, of comb structure, but to check whether certain idealizations made in the course of modeling are harmful or not. Third, I pointed out that optimization explanations can run into a serious underdetermination, in that one and the same geometrical structure can be the result of more than one kind of optimization. This is illustrated by the hexagonal 
tiling of the plane, which features in several optimization problems. Finally, I noted that it might be advantageous to take the construction process of optimal structures into account, because this leads to a more immediate confrontation with empirical data; it can be difficult to verify, or reject, purely mathematical optimization results. This results from a comparison of the first two with the second two explanations.

The hexagon turns out to be silent indeed. If the Acoustic Resonance Hypothesis is right, hexagons appear as soon as interferences disappear. However, this is not settled yet. The hexagon is also silent in that it refuses to tell us why exactly it is part of the honeycomb. It might be there because it is part of some kind of optimum - or for a different reason altogether.

\section{Acknowledgements}

I thank Claus Beisbart, Michael Esfeld, Hannes Leitgeb, Thomas Müller, Tilman Sauer, Raphael Scholl, and participants of the research colloquium AG Müller in Konstanz for comments on the paper at various stages of the writing process. This work was partially supported by the Swiss National Science Foundation, grant numbers 100011-124462/1 and 100018-140201/1, as well as by the Templeton World Charity Foundation through grant TWCF0078/AB4.

\section{References}

Baker, A. 2009. Mathematical Explanation in Science. British Journal for the Philosophy of Science 60(3): 611-633.

67.

. 2012. Science-Driven Mathematical Explanation. Mind 121(482): 243Synthese.

Baker, A. and M. Colyvan. 2011. Indexing and Mathematical Explanation. Philosophia Mathematica 19(3): 323-34.

Baron, S. 2014. Optimisation and mathematical explanation: doing the Lévi Walk. Synthese 191: 459-479.

Bauer, D. and K. Bienefeld. 2013. Hexagonal Comb Cells of Honeybees are Not Produced Via a Liquid Equilibrium Process. Naturwissenschaften 100: 45-49.

Bergman, D. J. and J. S. Ishay. 2007. Do Bees and Hornets Use Acoustic Resonance in Order to Monitor and Coordinate Comb Construction? Bulletin of Mathematical Biology 69: 1777-90. 
Cartwright, N. 1983. How the Laws of Physics Lie. Oxford, New York: Oxford University Press. Press.

Fejes Tóth, L. 1964. What the Bees Know and What they do not Know. Bulletin AMS 70: 468-81.

Frigg, R. and S. Hartmann. 2012. Models in Science. Http://plato.stanford.edu/entries/models-science/.

Glennan, S. 2002. Rethinking Mechanistic Explanation. Philosophy of Science 69(S3): 342-53.

Hales, T. C. 2000. Cannonballs and Honeycombs. Notices of the AMS 47(4): $440-9$.

- 2001. The Honeycomb Conjecture. Discrete and Computational Geometry 25: 1-22.

Hepburn, J. R., T. Muerrle, and S. E. Radloff. 2007. The cell bases of honeybee combs. Apidologie 38(3): 268-71.

Kadmon, J., J. S. Ishay, and D. J. Bergman. 2009. Properties of ultrasonic acoustic resonances for exploitation in comb construction by social hornets and honeybees. Physical Review E 79.

Klarreich, E. G. 2000. Foams and Honeycombs. American Scientist 88(2): $152-61$.

Lange, M. 2013. What Makes a Scientific Explanation Distinctively Mathematical? British Journal for the Philosophy of Science 64(3): 485-511.

Lyon, A. 2012. Mathematical Explanations Of Empirical Facts, And Mathematical Realism. Australasian Journal of Philosophy 90(3): 559-78.

Lyon, A. and M. Colyvan. 2008. The Explanatory Power of Phase Spaces. Philosophia Mathematica 16(2): 227-243.

Machamer, P., L. Darden, and C. F. Craver. 2000. Thinking about Mechanisms. Philosophy of Science 67(1): 1-25.

Norton, J. D. 2012. Approximation and Idealization: Why the Difference Matters. Philosophy of Science 79(2): 207-32.

Pierce, A. D. 1989. Acoustics. An Introduction to Its Physical Principles and Applications. New York: Acoustic Society of America.

Pincock, C. 2007. A Role for Mathematics in the Physical Sciences. Noûs 41(2): $253-75$. 
2012. Mathematics and Scientific Representation. Oxford, New York: Oxford University Press.

. 2014. Abstract Explanations in Science. British Journal for the Philosophy of Science .

Pirk, C. W. W., H. R. Hepburn, and S. E. Radloff. 2004. Honeybee combs: construction through a liquid equilibrium process? Naturwissenschaften 91(7): $350-3$.

Räz, T. 2013. On the Application of the Honeycomb Conjecture to the Bee's Honeycomb. Philosophia Mathematica 21(3): 351-60.

Rice, C. 2013. Moving Beyond Causes: Optimality Models and Scientific Explanation. Noûs .

Saatsi, J. 2011. The Enhanced Indispensability Argument: Representational versus Explanatory Role of Mathematics in Science. British Journal for the Philosophy of Science 62: 143-54.

Sternberg, S. 1994. Group theory and physics. Cambridge: Cambridge University Press.

Szpiro, G. G. 2003. Kepler's Conjecture. Hoboken, New Jersey: Wiley.

Tallant, J. 2013. Optimus prime: paraphrasing prime number talk. Synthese 190(12): 2065-83.

Weaire, D. and S. Hutzler. 1999. The Physics of Foams. Oxford: Clarendon Press.

Weaire, D. and R. Phelan. 1994. Optimal design of honeycombs. Nature 367(13): 123. 


\section{Figures}

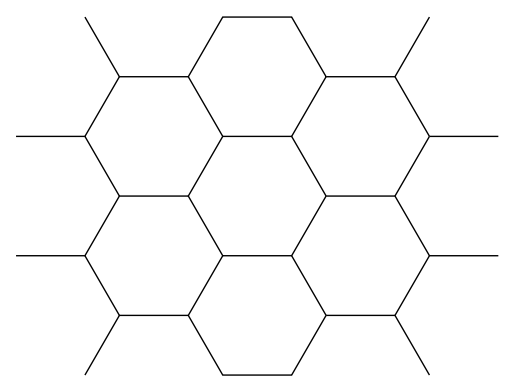

Figure 1: Hexagonal Tiling

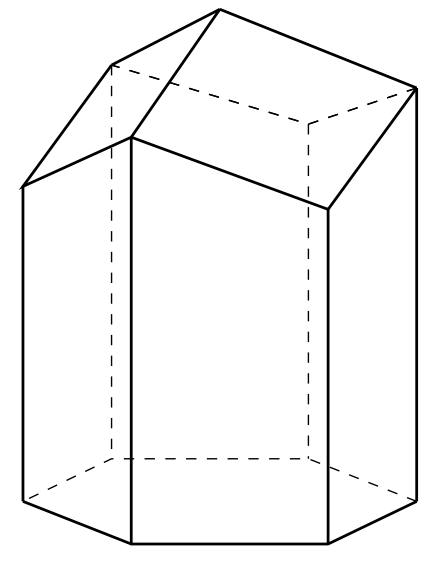

(a)

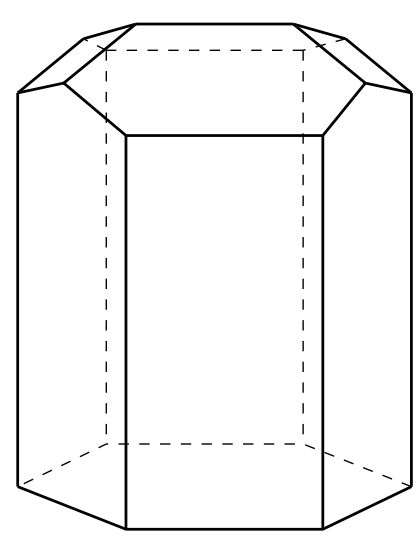

(b)

Figure 2: Actual Honeycomb (a) and Fejes Tóth Structure (b) 


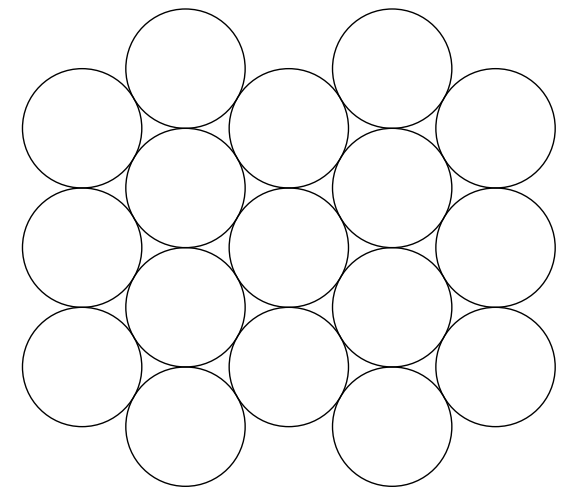

(a)

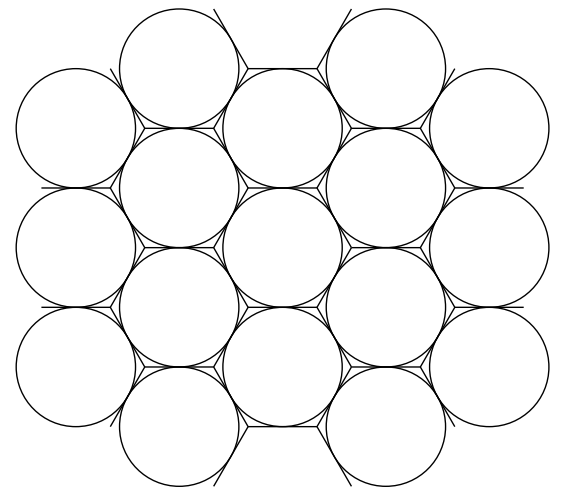

(b)

Figure 3: Close-Packing of Circles (a), corresponding Voronoi Cells with hexagonal tiling (b)

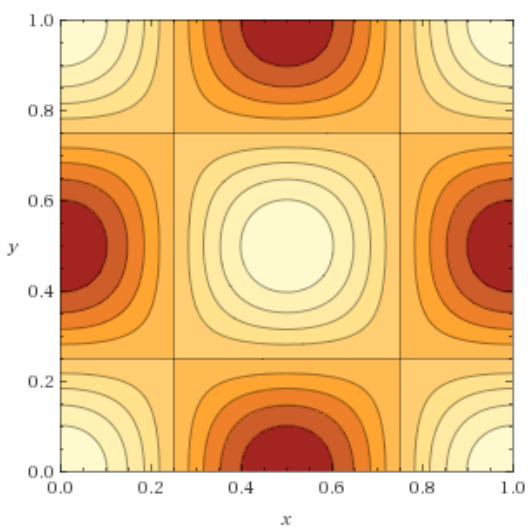

Figure 4: Room mode of a duct with unit square cross section. Dark areas signify low pressure. There are two straight lines in both $x$ and $y$ direction; these are nodes of constant pressure. 